VOL. $3(1970), 140$

\title{
Disturbance daily variation
}

\author{
H. F. Petersons
}

The first part of the thesis gives a review of earlier work on the geomagnetic disturbance daily variation. The disturbance daily variation is then computed for the year 1958 using data from fifty geomagnetic observatories distributed over both the northern and southern hemispheres of the Earth. Harmonic coefficients for the disturbance daily variation are computed and a spherical harmonic analysis using the weighted method of least squares is carried out.

Equivalent internal and external current systems which could give rise to the disturbance daily variation are then computed. Of particular interest is the result that the current systems are only approximately symmetrical about the geomagnetic equator and that the internal current systems although less intense have a similar pattern to the external current systems. The latter result suggests that the internal current systems are induced by the corresponding external current systems.

Finally the uniform conducting core model is investigated. A certain value for the conductivity and size of the conducting core was indicated for best agreement between the disturbance daily variation results and those from investigation of the geomagnetic quiet day variation.

Received 31 March 1970. Thesis submitted to the University of Sydney, May 1969. Degree approved, November 1969. Supervisor: Dr D.E. Winch. 